\title{
Mecanismos de Transposição Didática e Recontextualização da Computação para Educação Básica: uma investigação em experiências brasileiras
}

\author{
Ecivaldo de Souza Matos ${ }^{1}$, Euma Silva Santos ${ }^{1}$ \\ ${ }^{1}$ Instituto de Matemática e Estatística - Grupo de Pesquisa e Extensão Onda Digital \\ Universidade Federal da Bahia (UFBA) \\ Av. Adhemar de Barros, s/n, Ondina - Salvador - BA - Brasil \\ \{ecivaldo, euma.silva\} @ufba.br
}

\begin{abstract}
To be taught in schools, sciences go through processes such as Didactic Transposition and Recontextualization. This would be no different with Computer Science. In this work we have investigated in the literature how this has occurred in experiences in Basic Education. Both Didactic Transposition and Recontextualization are methodologies for adapting scientific knowledge to school knowledge, considering different aspects. In this paper we present some results of this investigation, especially the mechanisms of transposition and recontextualization of Computer Science used in Basic Education.
\end{abstract}

Resumo. Para serem ensinadas nas escolas, as ciências passam por processos como Transposição Didática e Recontextualização. Isso não seria diferente com a Computação. Neste trabalho investigamos na literatura como isso tem ocorrido em experiências de Computação na Educação Básica. Tanto a Transposição Didática quanto a Recontextualização são metodologias para adequação do conhecimento científico a conhecimento escolar, considerando diferentes aspectos. Neste artigo apresentamos alguns resultados dessa investigação, especialmente os mecanismos de transposição $e$ recontextualização de Computação são utilizadas na Educação Básica.

\section{Introdução}

No Brasil, a Computação ainda não é disciplina comum nos currículos da Educação Básica. O que observamos muitas vezes são instituições que incluem a informática em suas propostas curriculares, com o intuito de fomentar o uso de ferramentas e aplicativos de escritório pelos estudantes. Essa realidade se torna preocupante à medida que observamos que o uso e a variedade de tecnologias da informação têm crescido significativamente, tornando cada vez maior a necessidade do ensino de conteúdos de computação desde as séries iniciais da educação básica. Além disso, a computação tem se estabelecido como uma ciência, para além da sua natureza tecnológica, e que está presente nos mais diversos fenômenos do cotidiano; sendo, portanto, elemento fundamental na educação dos jovens escolares. 
Pensando nessa necessidade, muitas instituições mantêm projetos que visam trabalhar a Computação na Educação Básica. Nesse contexto, este estudo tem buscado identificar evidências da Transposição Didática (TD) e da Recontextualização da Ciência da Computação para Educação Básica. Sabemos que tanto a TD quanto a Recontextualização não são necessariamente aspectos de domínio formativo dos nossos professores de computação. Uma indicação disso é não terem sido encontrados, em nossa revisão de literatura, trabalhos que tratem explicitamente sobre o tema. Por isso, buscamos investigar se, ainda assim, encontramos ocorrência da TD ou da Recontextualização de Computação em experiências relatadas na literatura, mesmo que isso tenha ocorrido sem intencionalidade.

O estudo teve como metodologia um Mapeamento Sistemático de Literatura (MSL), com o objetivo de investigar (mapear) essas características em trabalhos que apresentem algum mecanismo (método ou técnica) de TD ou Recontextualização de Computação na Educação Básica como proposta metodológica.

Nas seções a seguir o estudo apresenta uma breve fundamentação conceitual e contextualização dos temas de investigação na Seção 2; descrição do processo de Mapeamento Sistemático, na Seção 3; alguns resultados na Seção 4; seguido de uma breve discussão desses resultados, na Seção 5.

\section{Fundamentação Teórica}

\subsection{Transposição didática e Recontextualização}

As práticas diárias de professores em ambiente escolar requer muitas habilidades. Nem sempre o conhecimento que construímos durante anos de experiência em uma formação acadêmica são suficientes para alcançarmos bons resultados na sala de aula. Isso tem muito a ver com a visão de Franco (2015) acerca da didática, o desafio de não poder prever a qualidade da aprendizagem em determinadas situações de ensino.

O saber a ensinar, segundo Alves-Filho (2000), é um processo resultante de uma característica metodológica chamada Transposição Didática (TD), em que o "saber sábio", sob o aspecto das "práticas sociais de referência", torna-se um conhecimento científico adequado para ser ensinado. Para Chevallard (1991), a TD é o trabalho metodológico de fazer de um objeto de saber a ensinar, um objeto de ensino. Para Alves-Filho (ibid), essa transformação passa por um processo que começa do saber científico para o saber a ensinar (transposição externa) e do saber a ensinar para o saber ensinado (transposição interna). Para Brockington \& Pietrocola (2005), a TD é processo metodológico com a finalidade de transformar um objeto de conhecimento em conhecimento aprendido.

\subsection{Recontextualização}

Na teoria da Recontextualização, Bernstein (1996, 1998), sociólogo britânico, propõe uma recontextualização do saber a partir do contexto social onde os indivíduos estão inseridos, isso inclui o tempo e o espaço.

Segundo Lopes (2005), é importante o estudo sobre os conceitos da recontextualização para entender os processos de reinterpretações (semioses) pelos quais passam os diferentes conteúdos durante sua circulação no ambiente educacional. 
Bernstein (1996, 1998), compreende que durante o processo de recontextualização os textos passam por uma fragmentação na passagem pelo corpo social da educação, alguns fragmentos são mais valorizados que outros, no entanto esses fragmentos são associados a outros fragmentos e têm a possibilidade de ressignificação (Lopes, 2005).

Para Mainardes \& Stremel (2010), o conceito da teoria da recontextualização foi desenvolvida no contexto da teoria do dispositivo pedagógico. O dispositivo pedagógico é o que Bernstein chama de regulador da comunicação pedagógica. $\mathrm{O}$ autor afirma que sua intenção era criar uma linguagem conceitual, onde fosse possível descrever a construção dos discursos e das práticas das relações pedagógicas, de forma que os contextos escolares fossem priorizados. Seria a pedagogização do conhecimento (Bernstein, 1998). Segundo Mainardes \& Stremel (2010) o modelo faz uma análise do processo pelo qual um conhecimento (rígido) é transformado em conhecimento escolar.

\subsection{Transp. Didática e Recontextualização em Computação: trabalhos correlatos}

Mesmo utilizando todas estratégias possíveis, percebemos diversas vezes dificuldades em apresentar conteúdos de computação para os alunos, sendo necessário todo conteúdo passar por uma adequação com o intuito de torná-lo mais "entendível" pelos estudantes. Estudos e relatos de experiências de outros professores apresentam essas mesmas dificuldades.

Nessa perspectiva entendemos que compreender e utilizar a Transposição Didática e a Recontextualização pode favorecer tanto os educadores quanto os educandos no ensino/aprendizagem de conteúdos de computação na Educação Básica. Para tal afirmação um estudo foi realizado com o objetivo de investigar situações onde a TD e a Recontextualização foram utilizadas em práticas escolares. Essas situações por muitas vezes foram praticadas de forma não intencional. No entanto, baseados em conceitos e características percebemos o quanto essas metodologias são comuns na sala de aula.

É muito comum ocorrências em que o professor/educador se apropria de diversas metodologias para facilitar a compreensão de conceitos. Romeike \& Göttel (2012), por meio da PBL (Problem Based Learning), transformam saber em exercícios e problemas, aspecto importante da TD. Alves et al. (2016), em seu relato, explicam como fizeram adequações dos conteúdos, de acordo com idade do aluno e o seu contexto social, outra característica da TD e da Recontextualização.

Baseado nos conceitos apresentados por Chevallard (1991), Alves-Filho (2000) e Achiam (2014), acerca da Transposição didática e por Bernstein (1998), Lopes (2005) e (Leite, 2007) concernentes à Recontextualização, elencou-se 12 características de TD e Recontextualização: 1 - Utilização de livros textos, com material organizado, hierarquizado, linearizado, gerando uma lógica sequencial; 2 - Transformações externas e internas; 3 - Relacionar os conteúdos com a cultura e o cotidiano dos estudantes; 4 Modernização e atualização do saber escolar e do saber a ensinar; 5 - Articular saber "velho" com "saber" novo; 6 - Transformar um saber em exercícios e problemas; 7 Tornar um conceito mais compreensível; 8 - Contexto primário (Regra distributiva); 9 Contexto recontextualizador (Regra de contextualização); 10 - Contexto Secundário (Regra de avaliação); 11 - "Que”. Classificação do grupo (Regra de reconhecimento); 12 - "Como". Enquadramento do grupo (Regra de realização). 


\section{Metodologia}

O objetivo deste estudo foi investigar quais mecanismos de Transposição Didática têm sido utilizados, intencional ou não, na Computação para Educação Básica. Nesta pesquisa realizamos um Mapeamento Sistemático de Literatura (MSL), conforme proposto por Kitchenham (2004).

Em conformidade com a proposta de Kitchenham (ibid), foram seguidas as seguintes etapas: (i) planejamento e definição das questões de pesquisa; (ii) aplicação dos critérios de inclusão e exclusão; (iii) execução do mapeamento e (iv) sumarização dos resultados.

\subsection{Definição de questões de pesquisa}

Para atender aos objetivos definidos, foram elaboradas uma questão principal (QP) e duas subquestões específicas (Sub-QP1, Sub-QP2).

Baseado no objetivo principal da pesquisa, o MSL tentou responder a seguinte questão de pesquisa:

QP - Quais mecanismos têm sido utilizados e relatados para Transposição Didática ou Recontextualização (intencional ou sem intencionalidade) de Computação para Educação Básica?

A questão principal foi dividida nas subquestões abaixo:

Sub-QP1 - Quais são os mecanismos implícitos ou explícitos de TD ou Recontextualização de Computação para Educação Básica?

- Rationale: a Sub-QP1 foi definida com o propósito de identificar estudos que utilizem TD ou Recontextualização de Computação para Educação Básica, apresentando modos e mecanismos de transposição utilizados.

- Hipótese: Durante as buscas nas bases de dados para realização deste MSL serão encontrados estudos ou relatos que apresentem modos e mecanismos de TD ou Recontextualização de Computação para Educação Básica.

Sub-QP2 - Quais são as similaridades entre os modos e mecanismos encontrados?

- Rationale: a Sub-QP2 foi definida com o propósito de identificar similaridades nos modos e mecanismos de TD ou Recontextualização de Computação para Educação Básica.

- Hipótese: Durante a análise dos resultados encontrados será possível identificar similaridades entre os modos e mecanismos de TD ou Recontextualização de Computação para Educação Básica coletados.

Dada a limitação de espaço, neste artigo apresentaremos apenas os resultados referentes à primeira subquestão de pesquisa (Sub-QP1).

\subsection{Critérios de inclusão e exclusão de estudos}

Foram incluídos os estudos que preencheram os seguintes critérios:

- estudos que apresentem método ou técnica de TD ou Recontextualização de Computação na Educação Básica. 
- estudos que foram publicados entre 2008 e 2017 (o mapeamento foi realizado em 2018).

Foram excluídos os estudos que preencheram pelo menos um dos critérios abaixo:

- artigos introdutórios para questões especiais e resumo de eventos;

- artigos duplicados;

- artigos sem acesso disponível para visualização e/ou download do arquivo, especialmente em casos em que os estudos são pagos ou não disponibilizados na biblioteca digital;

- artigos que não estejam veiculados em anais de conferência ou periódico;

- artigos que não se refiram a estudo ou práticas na educação básica.

\subsection{Processo de seleção de estudos e definição das palavras-chave e strings de busca}

A seleção ocorreu por meio da pesquisa em bibliotecas digitais e indexadores, de acesso livre e gratuito aos periódicos no âmbito da Universidade Federal da Bahia. A busca foi realizada em bases nacionais e internacionais. Todavia, neste momento, apresentaremos apenas os resultados referentes aos trabalhos nacionais.

No tocante à produção nacional, limitamos a busca aos veículos nacionais oficiais da SBC (Sociedade Brasileira de Computação) das comunidades de pesquisa em Educação em Computação e Informática na Educação, a citar: Portal de Periódicos da $\mathrm{CEIE}^{1}$ (Comissão Especial de Informática na Educação) e Anais do WEI ${ }^{2}$ (Workshop sobre Educação em Computação). Apresentamos a seguir, resumidamente, todo o processo do MSL.

Para isso, foi definida a seguinte string de busca:

- "transposição didática" AND ("ensino de computação" OR "educação básica" OR "aprendizado de computação" OR "aprendizagem de computação") AND ("escola básica" OR "educação básica" OR "ensino médio").

Alguns termos foram usados para provocar redundância, dado que alguns pesquisadores se referem à educação básica por meio de expressões distintas, idem para as diferenciações de significado entre as palavras "aprendizado" e "aprendizagem". Após a busca, com suporte da Mendeley ${ }^{3}$, deu-se seguimento às subetapas de execução do mapeamento ( $1^{\circ}$ filtro). Foram, portanto, aplicados os critérios de exclusão e inclusão. Em seguida, foram realizados os passos a seguir:

1. Seleção de estudos preliminares ( $1^{\circ}$ filtro: leitura de títulos, resumos e palavras-chave de cada artigo);

2. Seleção de estudos ( $2^{\circ}$ filtro: leitura de introdução e conclusão);

3. Leitura completa dos estudos selecionados na etapa 3 ;

4. Extração de dados dos estudos incluídos;

5. Tabulação e análise dos estudos selecionados restantes.

\footnotetext{
${ }^{1}$ https://www.br-ie.org/pub/index.php/

${ }^{2}$ https://sol.sbc.org.br/index.php/wei

${ }^{3}$ https://www.mendeley.com/
} 
Esse trabalho foi realizado por dois pesquisadores. Nas etapas 1 e 2, cada pesquisador apresentou os motivos de aceitação do artigo para os demais; em caso de discordância, esses pareceres foram submetidos a um professor expert (doutor na área, com mais de 05 anos de doutoramento) para validação.

Os artigos, cuja leitura da introdução e da conclusão (na etapa 2) não foram suficientes para o julgamento de aderência ao MSL, foram lidos na íntegra por meio das técnicas de leitura dinâmica, skimming e scanning, por não exigirem leitura precisa e detalhada do texto por completo.

Com a aplicação das strings, foram identificados 278 estudos. Dessa forma, foi aplicado o $1^{\circ}$ filtro de seleção, caracterizado pela leitura dos títulos e abstracts dos estudos, resultando em 38 trabalhos pré-selecionados.

O $2^{\circ}$ filtro aplicado, realizado de forma mais aprofundada, por meio da leitura de introdução e conclusão, resultou em 19 trabalhos selecionado, permitindo a realização da etapa final de leitura completa e extração de dados.

Dada a quantidade de trabalhos encontrados, não foi necessário estabelecer critérios de qualidade para classificação dos trabalhos selecionados.

\subsection{Extração de dados}

Para realização da extração de dados foram tomadas como base as características da TD e da Recontextualização apresentadas na Seção 2.3. A definição dessas características forneceu subsídios para responder as Sub-QP1 e Sub-QP2.

\subsection{Estudos selecionados}

Dentre os 19 estudos nacionais selecionados, três foram publicados pela Revista Brasileira de Informática na Educação (RBIE); três foram publicados no Simpósio Brasileiro de Informática na Educação (SBIE); seis no Workshop de Informática na Escola (WIE); um consta nos Anais dos Workshops do Congresso Brasileiro de Informática na Educação (WCBIE); e, por fim, seis foram apresentados no Workshop sobre Educação em Computação (WEI).

\section{Resultados}

A TD e a Recontextualização em suas conceitualizações formais exigem uma série de métodos que as formalizam. No entanto, observamos que por muitas vezes são utilizadas estratégias para ensinar Computação que se relacionam com as características da TD e Recontextualização estabelecidas na literatura. Tanto esses métodos quanto essas estratégias são utilizadas no intuito de promover resultados positivos no ensino e na aprendizagem escolar e, por isso, foram consideradas nesta investigação.

A partir dos dados extraídos, identificou-se uma ou mais características (vide Seção 2) em 18 dos 19 estudos deste MSL. Esse resultado indica que métodos e estratégias relacionadas à TD e à Recontextualização são utilizados para favorecer a compreensão de conteúdos da Ciência da Computação na Educação Básica. Isso fortalece as evidências de que a TD e a Recontextualização de Computação na Educação Básica são utilizadas, embora muitas vezes de modo não intencional. 
A característica mais frequente encontrada nos estudos é "7 - tornar um conteúdo mais compreensível". Em 12 dos 19 trabalhos, encontramos métodos e estratégias com tentativas de reduzir o grau de complexidade de conceitos, isso quer dizer que adequações foram realizadas. Amaral et al. (2015) na tentativa de facilitar o aprendizado de algoritmos e linguagem de programação a alunos do segundo ano do Ensino Médio, utilizou o ambiente Robocode (ambiente de simulação de batalhas entre robôs) como ferramenta de ensino. Rodrigues \& Lopes (2017) e Barbosa et al. (2015) utilizaram a Computação Desplugada como estratégia de inclusão da Computação na Educação Básica. Por sua vez, Wangenheim et al. (2014) utilizaram o Scratch. Todas essas múltiplas metodologias se apresentam como justificativas de facilitar conceitos (Alves-Filho, 2000).

A segunda característica mais presente nos estudos foi a 9, que aparece em sete artigos e trata do "Contexto recontextualizador". Essa característica se apoia tanto na Transposição Didática quanto na Recontextualização, visto que ela se refere a situações de ensino onde são realizadas adequações conforme a faixa etária do aluno, conteúdo e espaço escolar (Leite, 2007); e tem relação com a prática social de referência como aponta Astolfi (1995) apud Alves-Filho (2000).

Quadro 1. Visão geral das características encontradas por artigo

\begin{tabular}{|c|c|c|c|c|c|c|c|c|c|c|c|c|}
\hline \multirow{2}{*}{ Artigo } & \multicolumn{70}{|c|}{ Características da Transposição Didática e da } \\
\hline & $\mathbf{1}$ & $\mathbf{2}$ & $\mathbf{3}$ & $\mathbf{4}$ & $\mathbf{5}$ & $\mathbf{6}$ & $\mathbf{7}$ & $\mathbf{8}$ & $\mathbf{9}$ & $\mathbf{1 0}$ & $\mathbf{1 1}$ & $\mathbf{1 2}$ \\
\hline 1 & $\mathbf{x}$ & & & & $\mathbf{x}$ & & $\mathbf{x}$ & $\mathbf{x}$ & $\mathbf{x}$ & $\mathbf{x}$ & $\mathbf{x}$ & \\
\hline 2 & & & & $\mathbf{x}$ & & & & & & & & \\
\hline 3 & & & & & & & & & $\mathbf{x}$ & $\mathbf{x}$ & & $\mathbf{x}$ \\
\hline 4 & & & & & & & $\mathbf{x}$ & & & & & \\
\hline 5 & & $\mathbf{x}$ & & & & & $\mathbf{x}$ & & $\mathbf{x}$ & $\mathbf{x}$ & & \\
\hline 6 & & & & & & & $\mathbf{x}$ & & & & & \\
\hline 7 & & & $\mathbf{x}$ & & & $\mathbf{x}$ & $\mathbf{x}$ & & & $\mathbf{x}$ & & \\
\hline 8 & & & & & & & & $\mathbf{x}$ & & & & \\
\hline 9 & & & & & & $\mathbf{x}$ & $\mathbf{x}$ & & & & & \\
\hline 10 & & & & & $\mathbf{x}$ & & & & & $\mathbf{x}$ & & \\
\hline 11 & & & & & $\mathbf{x}$ & & $\mathbf{x}$ & & & & & \\
\hline 12 & & \multicolumn{1}{|c|}{ Nenhuma característica encontrada } & & \\
\hline 13 & & & & & & & $\mathbf{x}$ & & $\mathbf{x}$ & $\mathbf{x}$ & $\mathbf{x}$ & \\
\hline 14 & & & & & & $\mathbf{x}$ & & $\mathbf{x}$ & $\mathbf{x}$ & $\mathbf{x}$ & \\
\hline 15 & & & & & & $\mathbf{x}$ & $\mathbf{x}$ & $\mathbf{x}$ & $\mathbf{x}$ & $\mathbf{x}$ & \\
\hline 16 & $\mathbf{x}$ & & $\mathbf{x}$ & & & & $\mathbf{x}$ & & & & & \\
\hline 17 & & & $\mathbf{x}$ & & & $\mathbf{x}$ & $\mathbf{x}$ & & & & & $\mathbf{x}$ \\
\hline 18 & & & & & & & $\mathbf{x}$ & & & & & \\
\hline
\end{tabular}




\begin{tabular}{|l|l|l|l|l|l|l|l|l|l|l|l|l|}
\hline 19 & & & & & & $\mathbf{x}$ & & & $\mathbf{x}$ & & & $\mathbf{x}$ \\
\hline
\end{tabular}

Como exemplo de evidência dessa característica podemos citar os trabalhos de Wangenheim et al. (2014) e Alves et al. (2016). No primeiro, os autores afirmam que durante a execução da tarefa proposta houve divergência entre as duplas, sendo necessário recorrer a outras estratégias em que os alunos pudessem realizar a atividade individualmente. No segundo, os autores utilizaram conteúdos de História para ensinar Computação. Como os alunos eram de séries/anos diferentes, os conteúdos abordados diziam respeito ao currículo de cada faixa etária. O Quadro 1 apresenta uma visão geral das características encontradas.

\section{Discussão e considerações finais}

Em relação à Sub-QP1 (Quais são os mecanismos implícitos ou explícitos de TD e Recontextualização de Computação para Educação Básica?), foi possível identificar mecanismos de TD e Recontextualização a partir do mapeamento de suas características nos trabalhos selecionados.

Em nenhum dos trabalhos analisados foi identificado o uso da TD ou da Recontextualização de modo explicitamente intencional. Em 18 dos 19 trabalhos selecionados foram identificadas de uma a 7 características, de 12 ao total. Isso nos indica, considerando os limites dessa pesquisa, que no Brasil, a Transposição Didática e a Recontextualização de Computação têm sido utilizadas de forma não intencional no âmbito da Educação Básica.

Métodos e estratégias de TD e de Recontextualização funcionam quando inserimos às nossas práticas características pertinentes a elas. Os elementos elencados por Alves-Filho (2000) e por Leite (2007) e que definem respectivamente a TD e a Recontextualização são observadas com frequência no cotidiano do professor em ambiente escolar.

Utilização de recursos modernos, como por exemplo, documentos/materiais online, em vez de o tradicional livro/caderno, se caracteriza como uma atualização do saber a ensinar. Esse uso de novos recursos pode ser analisado como articulação do saber "velho" com o saber "novo", em que o "novo" esclarece melhor o antigo e o antigo atribui validade ao "novo".

Transformar um saber em exercícios e problemas é a regra mais importante e mais clara da TD, segundo Soares (2007). Ela está diretamente relacionada ao modo de avaliar e acompanhar a aprendizagem. O uso do Scratch, utilizado para ensino de programação em blocos, é um forte exemplo disso. Notamos ainda preocupação do professor em reduzir o grau de complexidade de determinados conceitos, isso é muito perceptível quando os autores relatam utilizar exemplos do cotidiano e da cultura do estudante para que esses conceitos sejam mais significativos para ele. Seria, por exemplo, eficaz utilizar o exemplo de uma rede de pesca para ensinar conceitos de Redes de Computadores em uma comunidade de pescadores?

Por fim, temos o discurso pedagógico defendido por Bernstein (1996) e que fundamenta a Recontextualização. Nesse discurso, o autor afirma que os saberes científicos são fragmentados enquanto passam pelo corpo educacional. Alguns desses 
fragmentos são mais valorizados em relação a outros e alguns são ressignificados. Isso funciona quando regulamos as condições que possibilitem o aprendizado (regras distributivas) e que "tal" conhecimento deve ser ensinado para determinado grupo. Todo conteúdo deve ser adequado por faixa etária (regra de recontextualização). É necessário que o discurso seja transformado em prática pedagógica (regra de avaliação). Os grupos devem ser classificados, reconhecidos (regra de reconhecimento) e enquadrados (regras de realização) possibilitando identificar as dificuldades e promover ações pertinentes ao aprendizado respeitando a singularidade de cada indivíduo.

Embora possamos afirmar o uso de métodos e estratégias de TD e Recontextualização de Computação na Educação Básica, é importante adotarmos o uso consciente dessas formas, considerando suas peculiaridades. Desse modo, temos uma metodologia a somar para o efetivo resultado esperado nas ações.

Como trabalhos futuros, vamos apresentar os demais resultados desta pesquisa, de modo a detalhar o funcionamento dos mecanismos de TC e Recontextualização encontrados nessa literatura. Também pretende-se investigar as similaridades entre os mecanismos apresentados, de modo a desenvolver insumos para a articulação epistemológica e metodológica com outras disciplinas da Educação Básica e da Educação Superior.

Por fim, pretende-se expandir esse mapeamento com relação ao período e ao universo de trabalhos, apresentando os resultados encontrados em bases científicas internacionais.

\section{Agradecimentos}

Os autores agradecem a Pró-Reitoria de Pesquisa, Criação e Inovação da Universidade Federal da Bahia e ao CNPq pelo apoio à pesquisa, por meio das bolsas de iniciação científica (ações afirmativas) e de produtividade em pesquisa.

\section{Referências}

Achiam, M. (2014). Didactic transposition: From theoretical notion to research programme. The biannual ESERA (European Science Education Research Association). Kappadokya, Turkey. [s/p]

Alves, N. C.; Wangenheim, C. G.; Rodrigues, P. E.; Hauck, J. C. R.; Borgatto, A. F. (2016). Ensino de Computação de Forma Multidisciplinar em Disciplinas de História no Ensino Fundamental - Um Estudo de Caso. Revista Brasileira de Informática na Educação, v. 24, n. 3.

Alves-Filho, J. P. (2000). Regras da transposição didática aplicadas ao laboratório didático. Cad. Cat. Ens. Fis., v.17, n. 2: p. 174-182.

Amaral, L. R., Silva, G. B., Pantaleão, E. (2015). Plataforma Robocode como Ferramenta Lúdica de Ensino de Programação de Computadores-Extensão Universitária em Escolas Públicas de Minas Gerais. Anais do XXVI Simpósio Brasileiro de Informática na Educação (SBIE). p. 200-208. 
Astolfi, J-P; Develay, M (1995). A Didática das Ciências. Papirus: Campinas.

Barbosa, A. V. S., Félix, A., Costa, L. S., Oliveira, R. N. R., Araújo, A. L. S. O., Costa, F. V. S. (2015). O ensino de conceitos computacionais para alunos de ensino médio: relato de experiência de uma gincana e das estratégias utilizadas pelos alunos na resolução das atividades desplugadas. Anais do XXIII WEI - Workshop sobre Educação em Computação. p. 120-129.

Bernstein, B. (1996). A estruturação do discurso pedagógico. Classe, códigos e controle. Petrópolis: Vozes.

. (1998). Novas contribuições de Basil Bernstein. Entrevista concedida a Mercê Espanya e Ramon Flecha. Rev. Bras. de Educ., Rio de Janeiro, n. 7, p. 88-88.

Brockington, G.; Pietrocola, M. (2005). Serão as regras da transposição didática aplicáveis aos conceitos de física moderna? Inv. em Ens. de Cienc. v.10, n.3, p. 387-404.

Chevallard, Y. (1991). La Transposicion Didactica: del saber sabio al saber enseñado. Argentina: La Pensée Sauvage.

Franco, M. A. S. (2015). Práticas pedagógicas de ensinar-aprender: por entre resistências resignações. Educ. Pesqui., São Paulo, v. 41, n. 3, p. 601-614, 2015.

Kitchenham, B. (2004). Procedures for performing systematic reviews. Keele, UK, Keele University, 33(TR/SE-0401), 28. http://doi.org/10.1.1.122.3308.

Leite, M. S. (2007) . Recontextualização e transposição didática. São Paulo: Junqueira \& Marin.

Lopes, A. C. (2005). Política de currículo: Recontextualização e Hibridismo. Currículo sem Fronteiras, v.5, n.2, p. 50-64.

Mainardes, J.; Stremel, S. (2010). A Teoria de Basil Bernstein e algumas de suas contribuições para as pesquisas sobre políticas educacionais e curriculares. Revista Teias. v. 11, n. 22, p. 31-54.

Rodrigues, A. N., Lopes, R. H. O. (2017). Proposta e adaptação de atividades desplugadas para o ensino de computação na educação básica. XXV Workshop sobre Educação em Computação (WEI). Anais do XXXVII Congresso da Sociedade Brasileira de Computação, p. 2227-2236.

Romeike, R. \& Gottel, T. (2012). Agile Project in High School Computing Education Emphasizing a Learners' Perspective. Proc. of 7th Work. Prim. Sec. Comp. Educ.

Wangenheim, C. G., Nunes, V. R., Santos, G. D. (2014). Ensino de Computação com SCRATCH no Ensino Fundamental - Um Estudo de Caso. Rev. Bras. de Inf. na Educ. (RBIE), v.22, n.3. 